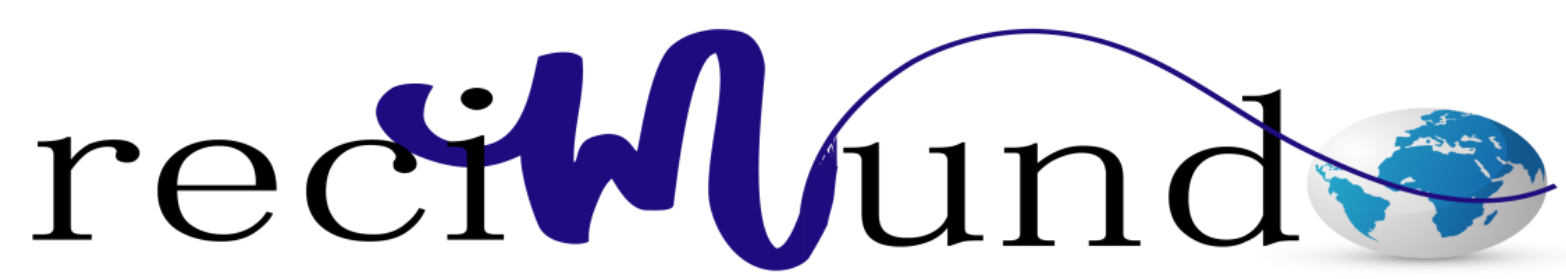

Revista Cientifica Mundo de la Investigación y el Conocimiento

María José Ortiz Zurita a ; Jazmín Jessenia Peñafiel León ${ }^{\text {b; }}$ Gabriela Natalia Torres Jara $^{c}$; Diana Valeria Delgado Campuzano ${ }^{\text {d; }}$ Evelyn Mariuxi Bautista Guaranda ${ }^{\mathrm{e}}$

Estudio conservacionista del bosque protector cerro Blanco (Ecuador) respecto a la experiencia del área de conservación privada Chaparrí (Perú)

Conservation study of the Cerro Blanco Protective Forest (Ecuador) regarding the experience of the Chaparri Private Conservation Area (Peru)

Revista Científica Mundo de la Investigación y el Conocimiento. Vol. 3 núm.3, septiembre, ISSN: 2588-073X, 2019, pp. 557-584

DOI: $10.26820 /$ recimundo/3.(3).septiembre.2019.557-584

URL: http://recimundo.com/index.php/es/article/view/538

Código UNESCO: 3106.01 Conservación

Tipo de Investigación: Artículo de Revisión

Editorial Saberes del Conocimiento

Recibido: 15/05/2019 Aceptado: 23/06/2019 Publicado: 30/09/2019

Correspondencia: mariajoseortizzurita@ hotmail.com

a. Magister en Administración de Empresas; Master Universitario en Dirección y Planificación de Turismo; Ingeniera en Administración de Empresas Turísticas y Hoteleras; Universidad Estatal de Milagro; Milagro, Ecuador; mariajoseortizzurita@ hotmail.com

b. Master en Gestión Turística; Ingeniera en Administración de Empresas Turísticas y Hoteleras; Universidad Estatal de Milagro; Milagro, Ecuador; jpnafiell@unemi.edu.ec

c. Ingeniera en Gestión Turística y Hotelera; Master Universitario en Gestión del Patrimonio Cultural y Museología; Universidad Estatal de Milagro; Milagro, Ecuador; gtorresj@unemi.edu.ec

d. Magister en Administración Ambiental; Ingeniera en Ecoturismo; Universidad Estatal de Milagro; Milagro, Ecuador; ddelgadoc@unemi.edu.ec

e. Licenciada en Turismo; Investigadora Independiente; Guayaquil, Ecuador; evymar92@gmail.com 


\section{Estudio conservacionista del bosque protector cerro Blanco (Ecuador) respecto a la experiencia del área de conservación privada Chaparrí (Perú)}

Vol. 3, núm. 3., (2019)

María José Ortiz Zurita; Jazmín Jessenia Peñafiel León; Gabriela Natalia Torres Jara; Diana Valeria Delgado Campuzano; Evelyn Mariuxi Bautista Guaranda

\section{RESUMEN}

Los espacios naturales, representan hoy en día para cualquier nación un medio que facilita el contacto con la naturaleza a toda la población en general y al mismo tiempo, los conduce a involucrarse en búsqueda de nuevas alternativas que aseguren su conservación y manejo, de esta manera, se cuidan sus características geográficas, fauna, flora, agua, clima y suelo entre otros. De allí, la aparición a nivel mundial de organizaciones públicas y privadas que tienen como propósito reconocer sus valores naturales y culturales; ejemplo de ello Ecuador, donde el Estado, comunidad, instituciones públicas u organizaciones privadas promueven la conservación del Bosque Protector Cerro Blanco ubicado en Guayaquil (Ecuador), es decir, conjugan su protección de sus s espacios e implementan actividades turísticas como estrategia para su proyección a nivel nacional, regional, local e internacional. En otras palabras, al poseer Ecuador al igual que los demás países en vía de desarrollo un potencial para atraer el mercado ecoturístico se convierte en una mirada hacia su naturaleza y así ofrecer nuevas experiencias. Ante estas consideraciones, surge el desarrollo de este artículo cuyo objetivo es plasmar estrategias para el manejo del Bosque Cerro Blanco (Ecuador) en base al estudio del área de Conservación Privada Chaparrí (Perú). Su metodología se identifica con las investigaciones documentales nivel descriptivo y lograr una adecuada valoración de los aportes bibliográficos que permitan plantear diferentes estrategias que promuevan el respectivo manejo del Bosque Protector Cerro Blanco, a fin de mantener su potencial en cuanto a la conservación de la flora, agua, suelo y fauna silvestre; su dinámica se cumple sobre la base del estudio del área de concentración Privada Charrí (Perú), es decir, mirar su posicionamiento como espacio natural y redimensionar nuevos conceptos que faciliten un manejo eficaz de este protector ambiental. Finalmente, se construyen las conclusiones.

Palabras Claves: Manejo protector Cerro Blanco (Ecuador); Área de conservación privada Chaparrí (Perú); Conservación; Espacio natural. 


\title{
Estudio conservacionista del bosque protector cerro Blanco (Ecuador) respecto a la experiencia del área de conservación privada Chaparrí (Perú)
}

Vol. 3, núm. 3., (2019)

María José Ortiz Zurita; Jazmín Jessenia Peñafiel León; Gabriela Natalia Torres Jara; Diana Valeria Delgado Campuzano; Evelyn Mariuxi Bautista Guaranda

\begin{abstract}
Natural spaces, today represent for any nation a means that facilitates contact with nature to the whole population in the general and at the same time, leads them to get involved in the search for new alternatives that ensure their conservation and management, of this way, its geographical characteristics, fauna, flora, water, climate and soil are taken care of, among others. Hence, the emergence worldwide of public and private organizations that aim to recognize their natural and cultural values; example of this Ecuador, where the State, community, public institutions or private organizations promote the conservation of the Cerro Blanco Protected Forest located in Guayaquil (Ecuador), that is, combine their protection from their spaces and implement tourism activities as a strategy for their projection at national, regional, local and international levels. In other words, by owning Ecuador as well as other developing countries, a potential to attract the ecotourism market becomes a glimpse into nature and new experiences are offered. In the face of these considerations, I raise the development of this article whose objective is to translate strategies for the management of Cerro Blanco Forest (Ecuador) based on the study of the area of Conservation Private Chaparrí (Peru). Its methodology is identified with the documentary research level descriptive and to achieve an adequate assessment of the bibliographic contributions that allow to propose different strategies that promote the respective management of the Cerro Blanco Protected Forest, to maintain its potential in terms of the conservation of flora, water, soil and wildlife; its dynamics are fulfilled on the basis of the study of the private concentration area Charrí (Peru), that is, to look at its positioning as a natural space and to resize new concepts that facilitate the effective management of this environmental protector. Finally, the conclusions are built.
\end{abstract}

Key Words: Protective handling Cerro Blanco (Ecuador); Private conservation area Chaparrí (Peru); Conservation; A natural space. 


\section{Estudio conservacionista del bosque protector cerro Blanco (Ecuador) respecto a la experiencia del área de conservación privada Chaparrí (Perú)}

Vol. 3, núm. 3., (2019)

María José Ortiz Zurita; Jazmín Jessenia Peñafiel León; Gabriela Natalia Torres Jara; Diana Valeria Delgado Campuzano; Evelyn Mariuxi Bautista Guaranda

\section{Introducción.}

La estructura productiva de Ecuador, se asienta en las actividades del sector primario; pero existe una necesidad para el Estado de generar nuevos ingresos, razón por la cual, impulsan nuevas iniciativas en el sector turístico, en donde las tendencias cambian debido a la presencia de un turista s exigente y comprometido con el cuidado y bienestar del medio ambiente, esto genera una estrategia turística exitosa; pero, introduce impactos en las comunidades locales, pobreza en el medio ambiente, por ello, se busca mediante la conservación de la herencia cultural e histórica mejorar la calidad de vida de cada uno de los actores involucrados, así, como proteger los espacios naturales de la actuación de los individuos hacia la fauna, flora, agua entre otro.

En consecuencia, el ecoturismo, actualmente representa para las naciones y en particular en Ecuador, una actividad importante que no sólo permite aumentar los ingresos; sino, que también ayuda a conservar aquellas áreas protegidas del país, visión nueva que conduce a reducir los efectos colaterales agregados por el abuso que incorporan algunas personas, instituciones u organizaciones en pro de atraer turistas, sin considerar su actuación en el medio ambiente. Ante estos hechos, se busca propiciar un cambio en la visión del turista, es decir, que sea más consciente, responsable de sus acciones, propiciador de un manejo adecuado de la naturaleza, en otras palabras, cuidarla, protegerla para la humanidad y así reducir los efectos negativos que puedan acarrear con su actuación.

Al respecto, la Unión Internacional para la Conservación de la Naturaleza (citado por Medina 2018) conceptualiza a la naturaleza de conservación como: 


\section{Estudio conservacionista del bosque protector cerro Blanco (Ecuador) respecto a la experiencia del área de conservación privada Chaparrí (Perú)}

Vol. 3, núm. 3., (2019)

María José Ortiz Zurita; Jazmín Jessenia Peñafiel León; Gabriela Natalia Torres Jara; Diana

Valeria Delgado Campuzano; Evelyn Mariuxi Bautista Guaranda

Aquella modalidad turística ambientalmente responsable consistente en viajar o

visitar áreas naturales con el fin de disfrutar y apreciar la naturaleza (así como

cualquier manifestación cultural del presente y del pasado), que promueve la

conservación, tiene bajo impacto de visitación y propicia un involucramiento activo

y socioeconómicamente benéfico de las poblaciones locales.

De acuerdo con lo citado, se puede entender que las zonas naturales caracterizadas por encontrarse en una ubicación de conservación o protección, representa un área destinada al disfrute con la naturaleza, donde los turistas deben respetar sus valores naturales y culturales, de esta manera, da apertura a la conjugación de los pueblos en pro de elevar las condiciones de sus espacios turísticos. Además, permite incrementar el ecoturismo como un medio que presenta capacidad para beneficiar a las comunidades que viven alrededor de las áreas protegidas, esto hace posible orientar y dirigir ese crecimiento con una propuesta sustentable que mejore el bienestar de las comunidades locales, minimice los impactos ambientales e impulse la industria turística.

En esta misma dirección, Medina (ob.cit) agrega que las áreas protegidas "son espacios geográficos claramente definidos, reconocidos y gestionados, mediante medios legales u otros tipos de medios eficaces para conseguir la conservación a largo plazo de la naturaleza y de sus servicios ecosistémicos y sus valores culturales asociados". (p. 8). Por lo tanto, concebir el ecoturismo desde la visión de las áreas protegidas, no es más que darle apoyo a su conservación, para mantenerlo como patrimonio natural y cultural, mediante los ingresos obtenidos como resultado de la venta de entradas, pago de impuestos locales, servicios entre otros medios que 


\section{Estudio conservacionista del bosque protector cerro Blanco (Ecuador) respecto a la experiencia del área de conservación privada Chaparrí (Perú)}

Vol. 3, núm. 3., (2019)

María José Ortiz Zurita; Jazmín Jessenia Peñafiel León; Gabriela Natalia Torres Jara; Diana Valeria Delgado Campuzano; Evelyn Mariuxi Bautista Guaranda

ayudan a resolver los costos que representa mantener la conservación de las tradiciones de un país.

De igual manera, se puede indicar que en la actualidad el Sistema Nacional de Áreas Protegidas representan aproximadamente el $20 \%$ del territorio nacional conservado, son parte del Patrimonio de Áreas Naturales del Estado (PANE), distribuidas en todo el territorio continental e insular, albergan una importante riqueza biológica y servicios eco sistémicos, de los cuales se benefician tanto las poblaciones urbanas como rurales, una riqueza paisajística que permite el turismo y la recreación en parte de ellas, y por su importancia ecológica trascienden fronteras que son reconocidas a nivel internacional. Por ello, en el 2020 Ecuador quiere convertir el turismo no es una actividad elitista ni excluyente, sino, en una actividad magníficamente incluyente que genera riqueza y espacios de equidad, para lo cual propone en su plan de desarrollo turístico estructurado por aquellas actividades desarrolladas en el país, que lo lleven a emprender e innovar de manera que el sector turístico sea competitivo con alta calidad, anfitriones capacitados, un entorno seguro, fluida conectividad y con un marco jurídico e institucional que promueva inversiones saludables.

En consideración a las ideas planteadas anteriormente, se puede indicar que en la provincia de Guayaquil, se encuentra el bosque protector Cerro Blanco, como una de las áreas protegidas, el mismo ofrece al turista la alternativa de distracción enfocada al deleite y aprecio a los recursos que la naturaleza brinda, para así generar en cada poblador y visitante la responsabilidad protegerlo. Es una reserva de 6.078 hectáreas del ecosistema bosque seco tropical de la costa ecuatoriana, ubicada en el extremo suroeste de la cordillera Chongón - 


\section{Estudio conservacionista del bosque protector cerro Blanco (Ecuador) respecto a la experiencia del área de conservación privada Chaparrí (Perú)}

Vol. 3, núm. 3., (2019)

María José Ortiz Zurita; Jazmín Jessenia Peñafiel León; Gabriela Natalia Torres Jara; Diana

Valeria Delgado Campuzano; Evelyn Mariuxi Bautista Guaranda

Colonche. Administrado por la Fundación Pro-Bosque, una institución jurídica de derecho privado, sin fines de lucro, creada bajo el Acuerdo Ministerial del Ministerio de Agricultura y Ganadería, el 9 de noviembre de 1992, con el objetivo de proteger y rehabilitar el bosque.

Al continuar con esta descripción, es importante destacar dentro de sus antecedentes históricos, en 1960 La Cemento Nacional (CN) compró el territorio. En 1989 CN se solicita al Ministerio de Agricultura y Ganadería para la protección de 2.025 hectáreas, parte del territorio fue otorgado en comodatos a la Fundación Natura. En 1990 se creó la infraestructura interpretativa y comenzó a recibir visitantes. Para el año 1992 se crea la Fundación Pro-Bosque (Organización Privada) para la administración del Bosque Protector Cerro Blanco. Posteriormente, en 2000 se extiende a 6.078 hectáreas, y una parte se encuentra en el perímetro urbano. En la actualidad, el Bosque Protector Cerro Blanco pertenece a la entidad privada HOLCIM (antes La CN), la cual es la encargada de financiar su desarrollo.

Según Ortiz, Penafiel, Torres, Delgado y Bautista (2019), destacaron que actualmente, HOLCIM busca que el Bosque Protector Cerro Blanco pueda auto gestionarse en todas sus operaciones, consideran que el turismo y sus actividades son una buena alternativa ya que compagina perfectamente con el cuidado del entorno natural y el involucramiento de la comunidad. Desde esta visión, se considera la capacidad del Bosque Protector Cerro Blanco para satisfacer la necesidad de los turistas y la búsqueda de su autogestión, surge la necesidad de plantear ideas que puedan ser aplicadas, mismas que deben estar enmarcadas en la Educación Ambiental debido a que este es uno de sus principales objetivos y es el marco legal bajo el cual se desarrollan sus actividades. 


\section{Estudio conservacionista del bosque protector cerro Blanco (Ecuador) respecto a la experiencia del área de conservación privada Chaparrí (Perú)}

Vol. 3, núm. 3., (2019)

María José Ortiz Zurita; Jazmín Jessenia Peñafiel León; Gabriela Natalia Torres Jara; Diana Valeria Delgado Campuzano; Evelyn Mariuxi Bautista Guaranda

Pese a que existen organismos privados que financian de forma parcial el mantenimiento y restauración del bosque, en esta dirección, Paz (2019), precisa que la reciente reducción del presupuesto del SNAP (organismo que gestiona las áreas protegidas) que disminuyó un 33,12\%, pasando de 6,7 millones de dólares a 4,5 millones de dólares; ocasiona problemas directos en entornos naturales como el Bosque protector Cerro Blanco, desequilibrando la economía interna del área e imponiendo la necesidad del incremento de sus fuentes alternas de ingresos, como las actividades deportivas y de entretenimiento de paga.

De acuerdo con este último planteamiento, se puede indicar que existe una necesidad de plasmar una propuesta en cuanto al manejo para el Bosque Protector Cerro Blanco, pero haciendo sus aportes desde la base del estudio del área de conservación Privada Chaparrí (Perú), con el fin de compaginar una actuación conservacionista por la comunidad. Tal como lo refleja el Plan Maestro de Conservación del área de Chaparrí, donde la comunidad Santa Catalina de Chongoyape, de manera consciente, ha planteado como estrategia de administración sus recursos naturales, apostar por la conservación y uso sustentable de estos, como la mejor alternativa para aliviar la pobreza en el largo plazo, contribuir a la conservación de diferentes especies de flora y fauna silvestre amenazadas. Esto con la firme esperanza de que constituyan una alternativa para el beneficio socioeconómico de la población dentro de su comunidad. Es decir, llevar a cabo proyectos de reintroducción y conservación con el consentimiento y participación de comuneros que valorizan sus recursos naturales.

Según las apreciaciones dadas, se puede decir que el desarrollo del presente artículo busca plasmar una propuesta en cuanto al manejo del Bosque protector Cerro Blanco (Ecuador) 


\section{Estudio conservacionista del bosque protector cerro Blanco (Ecuador) respecto a la experiencia del área de conservación privada Chaparrí (Perú)}

Vol. 3, núm. 3., (2019)

María José Ortiz Zurita; Jazmín Jessenia Peñafiel León; Gabriela Natalia Torres Jara; Diana

Valeria Delgado Campuzano; Evelyn Mariuxi Bautista Guaranda

sobre la base del área de conservación privada Chaparrí (Perú), con el fin de compaginar sus criterios y hacer posible la introducción de nuevas acciones que aseguren no sólo los ingresos económicos, sino, mantengan elevada las condiciones naturales, culturales y ambientales que caracterizan a la respectiva área protegida. Para lo cual, se hace necesario establecer la metodología como fuente que propicia las condiciones reales para su desarrollo posterior.

\section{Método.}

La acción investigativa, para lograr cambios progresivos en la estructura científica necesita de un actuar en correspondencia con la naturaleza metodológica, por lo tanto, para encaminar dicho evento, es importante agregar lo citado por Pérez (2019) “el método es el medio viable que le dice al investigador los pasos a seguir en un determinado momento de la búsqueda de nuevas informaciones”. (p.71). En consecuencia para lograr, efectividad durante la respectiva seleccionar de las actividades propuestas a fin de mejorar las condiciones ambientales, valores y cultura que hacen vida en el Bosque Protector Cerro Blanco, se estima incorporar una herramienta viable que sea capaz de alinear cada fase por cumplir.

En este orden de ideas, Pérez (ob.cit), destaca que para lograr eficiencia en una respuesta investigativa de apoyo bibliográfico "es indispensable el manejo del método de contingencia, “empleado para determinar el grado de asociación entre dos caracteres o dos serie de datos”.(p. 78). Lo planteado, lleva a entender que, al tener como interés científico plasmar una propuesta de manejo para el bosque protector Cerro Blanco, en base al estudio del área de conservación privada Chaparrí (Perú), y ubicarla de manera correspondiente, este método le asegura al investigador el reconocimiento de ideas previas que al ser compaginas, facilitan la construcción 


\section{Estudio conservacionista del bosque protector cerro Blanco (Ecuador) respecto a la experiencia del área de conservación privada Chaparrí (Perú)}

Vol. 3, núm. 3., (2019)

María José Ortiz Zurita; Jazmín Jessenia Peñafiel León; Gabriela Natalia Torres Jara; Diana Valeria Delgado Campuzano; Evelyn Mariuxi Bautista Guaranda

científica manteniendo un orden en cuanto a los criterios científicos que deben ser debidamente conjugados.

\section{Tipo de Investigación}

El recorrido metodológico que caracteriza a este artículo se encuentra insertado en la investigación documental, vista por Barrientos (2019) aquella que permite "obtener conocimientos que parten del análisis de datos, ya sea recolectados o analizados de otras investigaciones y se extraen de distintas fuentes de consulta". (p.56). Es decir, mediante su ubicación en el marco de la investigación documental, se puede indicar que, el investigador realiza diferentes actividades vinculadas con la revisión de documentos importantes estrechamente relacionados con la temática, para analizarlos y extraer de ellos sus apreciaciones generales que dan cabida a la construcción del cuerpo teórico del artículo.

Asimismo, se puede complementar que debido al propósito que presenta el desarrollo del artículo, es importante ubicarla en un nivel descriptivo, pues, mediante esta apreciación metodológico, el investigador puede, no sólo extraer ideas, sino relacionarlas mediante indagaciones profundas que le ayuden a compaginarlas para finalmente formular nuevos criterios que se conviertan en aportes significativos para el desarrollo de posibles cambios, en este particular, la propuesta de manejo para el bosque protector Cerro Blanco (Ecuador) en base al estudio del área de conservación privada Chaparrí (Perú). Apoyado este planteamiento, en Arias (2011) la investigación descriptiva consiste en "la caracterización de un hecho, fenómeno, individuo o grupo, para establecer su estructura o comportamiento. Los resultados de este tipo de 


\section{Estudio conservacionista del bosque protector cerro Blanco (Ecuador) respecto a la experiencia del área de conservación privada Chaparrí (Perú)}

Vol. 3, núm. 3., (2019)

María José Ortiz Zurita; Jazmín Jessenia Peñafiel León; Gabriela Natalia Torres Jara; Diana Valeria Delgado Campuzano; Evelyn Mariuxi Bautista Guaranda

investigación se ubican en un nivel intermedio en cuanto a la profundidad de los conocimientos se refiere". (p.24)

\section{Técnicas Documentales}

El proceso científico para lograr los objetivos previamente trazados, requiere de incorporar técnicas de revisión bibliográficas que le ofrezcan diferentes oportunidades para recolectar e interpretar las ideas expuestas en otros documentos bibliográficos. En tal sentido, Barrientos (ob.cit) las considera "como medios de apoyo para la recopilación de informaciones especiales que le aseguran al lector un campo abierto de oportunidades reflexivas".(p.51). De allí, que su incorporación hace posible no sólo revisar los documentos, sino extraer aquellos planteamientos de interés para la conducción crítica del tema a tratar.

\section{Técnicas de Recolección de Información}

Una vez seleccionada las técnicas documentales, es importante incorporar durante el acopio de los aportes bibliográficos el uso de otros medios, capaces de complementar el trabajo, en pro de generar nuevas apreciaciones generales y competentes debidamente vinculadas con el tema seleccionado. Para Arias (ob.cit) “este proceso no es más que la combinación de herramientas didácticas competentes que ayuden al investigador a lograr el respectivo abordaje del tema, de manera ordenada y coherente a las características del fenómeno en estudio.’(p.32).

Este planteamiento, permite resaltar el significado que posee la introducción de diferentes técnicas documentales, para así mantener la respectiva correspondencia con el tipo de investigación, además, de ubicar su relación con el método, en este artículo, es importante 


\section{Estudio conservacionista del bosque protector cerro Blanco (Ecuador) respecto a la experiencia del área de conservación privada Chaparrí (Perú)}

Vol. 3, núm. 3., (2019)

María José Ortiz Zurita; Jazmín Jessenia Peñafiel León; Gabriela Natalia Torres Jara; Diana Valeria Delgado Campuzano; Evelyn Mariuxi Bautista Guaranda

agregar la lectura rápida como una forma de seleccionar los cuerpos teóricos de interés, además, de la revisión interpretativa como herramienta encargada de ofrecer nuevas sugerencias que faciliten la comparación entre los elementos presente en el problema de investigación.

\section{Resultados.}

Los aspectos vinculantes con los aportes que la investigación, busca ofrecer al lector, se encuentran una serie de argumentos previamente seleccionados y analizados con el fin de comprender sus apreciaciones y llegar a generar nuevas ideas capaces de proyectar su contenido hacia valoraciones flexibles y coherente a la naturaleza del estudio. Los mismos, se presentan a continuación:

\section{Manejo Protector del Bosque Cerro Blanco}

El Bosque Protector Cerro Blanco es considerado el lugar ideal para la implementación del proyecto de compensación de huella de carbono donde el Banco de Desarrollo de América Latina (CAF) a través de la Fundación Pro-Bosque por ser uno de los remanentes mejor conservados de bosque seco de la costa ecuatoriana y uno de los pulmones verdes de Guayaquil, señaló Bernardo Requena, director representante de CAF en Ecuador. Destacó asimismo que esta actividad de compensación de huella de carbono busca generar consciencia sobre el impacto ambiental y pretende convertirse en una iniciativa que pueda ser replicada.

Los árboles fueron plantados en una extensión de 13 hectáreas y pertenecen a 15 especies forestales (entre ellas: guachapelí, ceibo macho, cascol, amarillo, laurel, cocobolo, bálsamo, caoba, colorada, guayacán, pechiche y ébano); logrando de esta manera fortalecer el proceso de 


\section{Estudio conservacionista del bosque protector cerro Blanco (Ecuador) respecto a la experiencia del área de conservación privada Chaparrí (Perú)}

Vol. 3, núm. 3., (2019)

María José Ortiz Zurita; Jazmín Jessenia Peñafiel León; Gabriela Natalia Torres Jara; Diana

Valeria Delgado Campuzano; Evelyn Mariuxi Bautista Guaranda

regeneración natural del bosque y el enriquecimiento del mismo a través de la incorporación en cantidad y diversidad de especies forestales propias de este ecosistema, que naturalmente tardarían mucho tiempo en llegar y establecerse. Muchas de estas especies constituyen fuente de alimento y enriquecen el hábitat del Papagayo de Guayaquil (Ara ambiguus guayaquilensis), ave símbolo natural de dicha ciudad que se encuentra en peligro de extinción y que tiene una estrategia de conservación en implementación para evitar que su población siga reduciéndose.

La estrategia ambiental regional de CAF, reconoce al componente de medio ambiente como determinante en la transformación productiva y desarrollo de los países latinoamericanos. Por esta razón, esta actividad de compensación de huella de carbono, busca generar conciencia sobre el impacto ambiental que genera una persona o una institución, a través de las emisiones de dióxido de carbono (CO2) en sus actividades cotidianas, y pretende convertirse en un modelo que se replique en diversas instituciones del país. Esta actividad permitirá compensar 189 toneladas de $\mathrm{CO} 2$, de las 229 toneladas que fueron generadas por la oficina de CAF en Ecuador.

Cabe agregar que, el Bosque Protector de Cerro Blanco comprende un área de bosque seco tropical, donde se conservan más de 700 especies de plantas vasculares, 220 especies de aves, 54 especies de mamíferos, 22 especies de reptiles, 8 de anfibios y muchas especies de insectos. Este tipo de hábitat se puede encontrar en las provincias de Manabí, Los Ríos, El Oro, Loja y Guayas. En la capital de esta última provincia, Guayaquil, solo subsisten siete bosques secos y Cerro Blanco encabeza la lista por ser el más vasto. No obstante, la actuación de los individuos, crean condiciones ambientales en el mismo, caracterizada por el deterioro progresivo de sus espacios. 


\section{Estudio conservacionista del bosque protector cerro Blanco (Ecuador) respecto a la experiencia del área de conservación privada Chaparrí (Perú)}

Vol. 3, núm. 3., (2019)

María José Ortiz Zurita; Jazmín Jessenia Peñafiel León; Gabriela Natalia Torres Jara; Diana Valeria Delgado Campuzano; Evelyn Mariuxi Bautista Guaranda

Figura $\mathbf{N}^{\circ} 1$ Tala Ilegal en el Bosque Protector Cerro Blanco

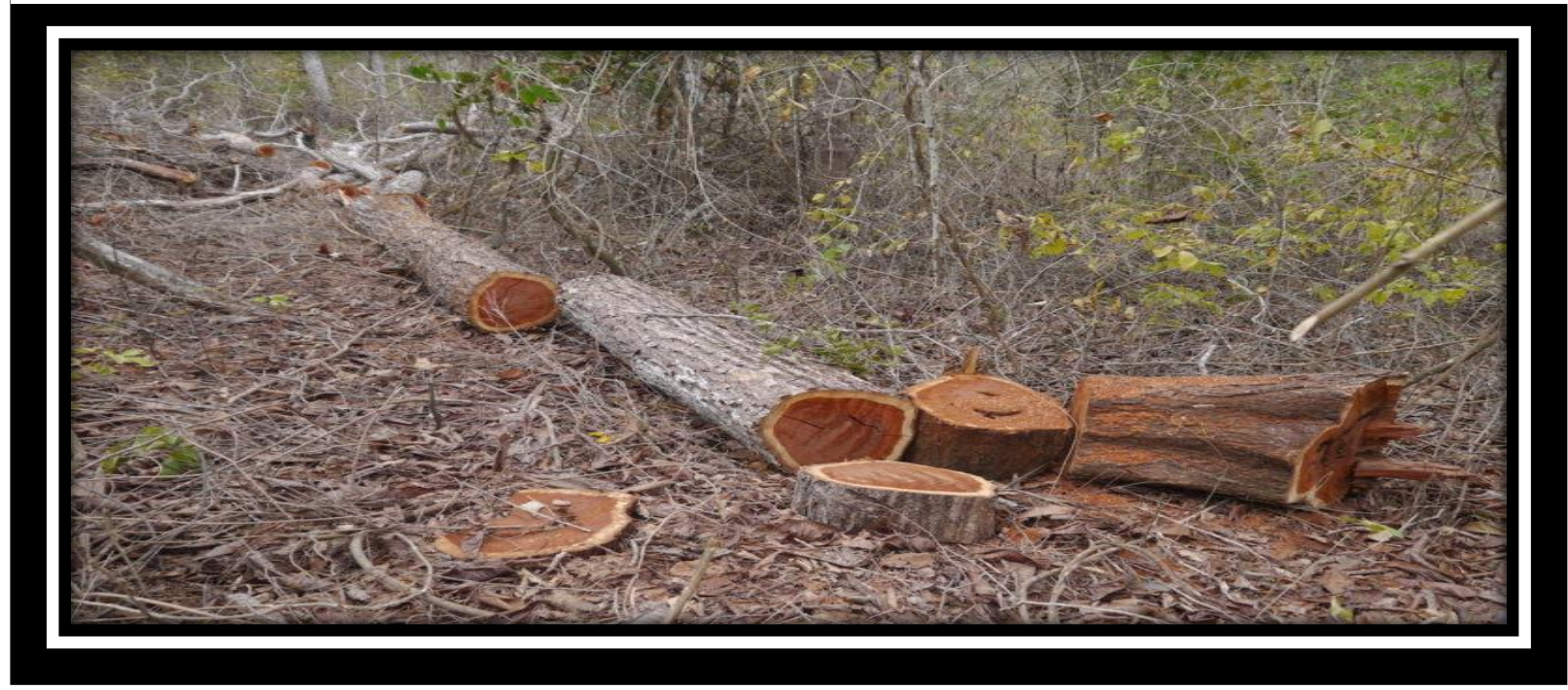

Fuente: Fundación Pro-Bosque (2018)

Es importante resaltar que, estas actuaciones inadecuadas, llevan a entender que el número de guardabosques llega a 12 y aunque la demanda es mayor, esa es la cantidad que la Fundación Pro-Bosque puede cubrir. A pesar de no llevar un registro de las incidencias por tala o cacería. Los guardabosques calculan que al menos 10 veces por año se han topado con incidentes, pero muy aparte de eso, también se encuentran con huellas, tarimas, trillos, que son muestra de actividades ilícitas.

Si en 1989 no se hubiese hecho la petición de que se declaren 2075 hectáreas como bosque protector, Cerro Blanco sería impensable. Es más, actualmente se hablaría de cómo el crecimiento inmobiliario acabó con uno de los relictos de bosque seco, y no se podría observar cómo ese espacio natural se aviva de forma sorprendente cada invierno. Pero ese terreno se 


\section{Estudio conservacionista del bosque protector cerro Blanco (Ecuador) respecto a la experiencia del área de conservación privada Chaparrí (Perú)}

Vol. 3, núm. 3., (2019)

María José Ortiz Zurita; Jazmín Jessenia Peñafiel León; Gabriela Natalia Torres Jara; Diana Valeria Delgado Campuzano; Evelyn Mariuxi Bautista Guaranda

recuperó y entró a ser parte del 42,9 \% de bosques protectores gestionados por actores privados.

Desde entonces, el camino recorrido para mantenerse ha tenido buenos momentos asociados a su conservación, pero también instantes en que este ecosistema ha estado en peligro, como ha resultado la tala ilegal que amenaza al bosque más grande de Guayaquil

Si se habla en cifras, el Cerro Blanco ha logrado expandirse a 6078 hectáreas y que, entre los reconocimientos recibidos, conste el premio Latinoamérica Verde 2016 en la categoría bosque y flora. Pero estos logros no han sido sencillos, sobre todo cuando a lo largo de su frondosa vegetación se esconde más de una amenaza. Y es que su ubicación geográfica y los recursos naturales que preserva lo han vuelto atractivo principalmente para algunos negocios poco sostenibles.

Cabe agregar, que el Cerro Blanco se encuentra en el Km 16 de la Vía a la Costa. Aquí, las zonas verdes han quedado rezagadas para los parterres y plantas ornamentales de urbanizaciones y centros comerciales: Palmeras, árboles pequeños y explanados de estacionamientos, son usuales. El cartel que anuncia el bosque apenas se distingue entre la vegetación que sus paredes encierra. Es una 'zona franca' donde el disfrute es la naturaleza. Para ser exactos, este bosque es de las pocas conexiones que le resta a Guayaquil con la Cordillera Chongón Colonche, que a atraviesa tres provincias recorriendo $95 \mathrm{Km}$ de la costa ecuatoriana y formando un cinturón de bosques secos.

En definitiva, el potencial ambiental de Cerro Blanco es alto, pero sus riesgos también. Además de convivir con la expansión citadina, riñe lindero a lindero con la extracción de materiales: otro negocio bastante rentable en los alrededores. Es decir, este trecho de selva vive 


\section{Estudio conservacionista del bosque protector cerro Blanco (Ecuador) respecto a la experiencia del área de conservación privada Chaparrí (Perú)}

Vol. 3, núm. 3., (2019)

María José Ortiz Zurita; Jazmín Jessenia Peñafiel León; Gabriela Natalia Torres Jara; Diana Valeria Delgado Campuzano; Evelyn Mariuxi Bautista Guaranda

en medio de un boom económico. Por ello, es importante destacar que a inicios de 2017 una cantera traspasó los límites del bosque protector y se inició una querella. Los involucrados fueron Fundación Pro-Bosque, creada entre actores privados para administrar Cerro Blanco, y el yacimiento de roca caliza Evadriana VI. El conflicto lo resolvió el MAE, que al tratarse de áreas particulares interviene desde lo legal y técnico. El alcance de esta irrupción fue de 20. 000 metros cuadrados según la Agencia de Regulación y Control Minero (ARCOM). Finalmente, la cantera fue suspendida.

Posteriormente, se tuvo que lidiar con otro amenaza a finales de 2016, el problema lo desató Casas Viejas, una comuna de cerca de 300 viviendas que colinda con el bosque y que reclamaban hasta cinco hectáreas del lugar; luego, ese mismo año, a un hombre se le ocurrió lotizar parte del territorio de Cerro Blanco, repartirlo a agricultores y dejar que éstos introduzcan cultivos de maíz. Todos los intentos fueron interrumpidos en primera instancia por los guardabosques y después, con registros del MAE o la Policía Ambiental para crear precedentes.

De este último episodio la Unidad de Protección del Medio Ambiente (UPMA) de la Policía Nacional levantó un informe: "Se evidencia especies de flora entre árboles y arbustos talados de diferentes diámetros como: chirigua, compoño, guasmo, beldaco, ceibo, guayacán, amarillo, palo santo, colorado. Esta vegetación habría sido talada en días anteriores por personas que presuntamente están limpiando para realizar cultivos, en una extensión de aproximadamente tres hectáreas, las mismas que aparentemente se encuentran dentro del bosque protector Cerro Blanco. Los sectores del bosque que suelen ser vulnerados escapan a la vista del turista común, pues, el espesor de la vegetación o por la lejanía de sus fronteras. Considerando que al norte se 


\section{Estudio conservacionista del bosque protector cerro Blanco (Ecuador) respecto a la experiencia del área de conservación privada Chaparrí (Perú)}

Vol. 3, núm. 3., (2019)

María José Ortiz Zurita; Jazmín Jessenia Peñafiel León; Gabriela Natalia Torres Jara; Diana

Valeria Delgado Campuzano; Evelyn Mariuxi Bautista Guaranda

localizan propiedades privadas y el Bosque Papagayo; al sur se hallan terrenos de Holcim y la Vía a la Costa; al este está el Bosque Protector Prosperina; y al oeste limita con bienes privados y la Cordillera Chongón Colonche.

No obstante a pesar de todos estos procesos violentos realizados por la comunidad hacia el Bosque Protector Cerro Blanco, se puede encontrar en sus espacios naturales Cerro Blanco tiene un camino de tierra que conduce hacia un lugar denominado centro de visitantes, donde según la resistencia física se puede escoger entre tres senderos: Buena Vista, Higuerón y Mono Aullador. A medida que se avanza por las rutas elegidas, el caminante se topa con matorrales apiñados y vegetación que se enreda alrededor de una serie de puentes de madera. Al llegar a la llanura, aparte de un espacio de meditación, hay un mirador y desde allí, se observa a un gavilán que revolotea en círculos buscando su presa. La naturaleza es una exhibición sin descanso.

Ante estos hechos, los expertos que trabajan con Pro-Bosque, visualizan la necesidad urgente de llevar a cabo nuevas acciones que den pauta para la conservación, es decir, establecer nuevos corredores biológicos para ecosistemas de la Costa ecuatoriana, de este modo, sería la Chongón Colonche con Bosque Protector Cerro Blanco y Bosque Protector Prosperina que se conecte con Bosque Protector Cerro Paraíso, hasta el sector cercano a unos condominios por allí. El costo de esta propuesta es alta, por eso el ambientalista señala que el proyecto necesita de más actores. Por ello, la Municipalidad de Guayaquil debe trabajar en conjunto con el Ministerio de Ambiente Ecuador y la sociedad civil en la implementación y educación, se necesita formar y crear socios estratégicos, para así, dar camino finalmente es la conexión permanente con sitios cercanos igual de diversos para que la naturaleza se amplíe y no retroceda a pequeños espacios. 


\section{Estudio conservacionista del bosque protector cerro Blanco (Ecuador) respecto a la experiencia del área de conservación privada Chaparrí (Perú)}

Vol. 3, núm. 3., (2019)

María José Ortiz Zurita; Jazmín Jessenia Peñafiel León; Gabriela Natalia Torres Jara; Diana Valeria Delgado Campuzano; Evelyn Mariuxi Bautista Guaranda

De acuerdo con lo citado por Ortiz, Penafiel, Torres, Delgado y Bautista (ob.cit), cerro

Blanco es un bosque seco tropical, en donde las temperaturas llegan a ser mayores o iguales a $24^{\circ}-26^{\circ} \mathrm{C}$ con una precipitación anual entre 800 a $1200 \mathrm{~mm}$. Se ve influenciado por la corriente marina de Humboltd que bordea las costas del Pacífico que vienen desde el Sur hacia el Norte causando desertificación en zonas continentales cercanas por su naturaleza fría. A toda esta área como ya se ha mencionado se la conoce como Eco-región Tumbesina en la cual está ubicada el Bosque Protector Cerro Blanco, designada cono área de importancia para las aves entre 1999 y 2000.

Asimismo, se precisa que el bosque protector Cerro Blanco es uno de los más grandes y mejor conservados fragmentos del bosque seco tropical ecuatoriano. Ofrece una de las mejores manifestaciones de supervivencia de tal vez 100 de las más de 500 especies de plantas vasculares registradas, que son endémicas de la región del bosque seco tropical. Algunas de las especies de árboles que encontramos en el bosque son: árboles maderables: guachapelí o Albizia, guachapele, guayacán, pechiche, colorado. Árboles no maderables: ceibo, bototillo o Cochlospermun, vitifolium, balsa.

En este mismo sentido de argumentación, los autores antes señalados, precisaron que el ingreso al Bosque Protector Cerro Blanco tiene los siguientes costos: adultos \$4, niños \$3 y personas de la tercera edad \$2. De acuerdo a la oferta que poseen enlistamos sus productos: senderismo, guía para observación de aves, campamentos y otros atractivos y servicios tales como área infantil, de descanso, meditación, picnic, mirador Buena Vista - glorieta con hamacas 


\section{Estudio conservacionista del bosque protector cerro Blanco (Ecuador) respecto a la experiencia del área de conservación privada Chaparrí (Perú)}

Vol. 3, núm. 3., (2019)

María José Ortiz Zurita; Jazmín Jessenia Peñafiel León; Gabriela Natalia Torres Jara; Diana

Valeria Delgado Campuzano; Evelyn Mariuxi Bautista Guaranda

y charlas sobre temas de conservación con técnicos especializados. Aspectos significativos para su valoración como un potencial eco turista para Ecuador.

\section{Área de Conservación Privada Chaparrí (Perú)}

Con el fin de compaginar, los elementos relevantes que estructuran al Bosque Protector Cerro Blanco y encontrar alternativas viables para repotenciar su capacidad de expansión, se hace necesario reconocer el área de conservación privada Chaparrí (Perú) como un elemento esencial para replantearse nuevos enfoques de importancia en su desarrollo como espacio natural turístico.

De lo antes indicado, se puede considerar lo planteado por Ortiz, Penafiel, Torres, Delgado y Bautista (ob.cit), en cuanto al Área de Conservación Privada Chaparrí se encuentra ubicada en el límite sur de la ecorregión denominada Bosque Seco ecuatorial, su ubicación es considerada estratégica debido a que tiene cercanía con áreas protegidas por el estado como el Santuario Histórico Bosque de Pomac y la zona reservada de Laquipampa, de esta manera Chaparrí sirve como un corredor biológico entre estas dos áreas. Tiene una extensión de 34.312 h. y se ubica dentro de territorio comunitario "Santa Catalina de Chongoyape" cuya extensión es de 41.260h en la Provincia de Chiclayo, Departamento de Lambayaque.

Esta reserva tuvo origen gracias a que las comunidades campesinas recibieron grandes extensiones de terreno después de la reforma agraria por la que pasó Perú en el año 69, la propuesta para que sea un Área de Conservación Privada fue dada por el Sr. Heinz Plenge, actual director de La Huerta de Chaparri, así se inició el trabajo en conjunto con la comunidad para 


\section{Estudio conservacionista del bosque protector cerro Blanco (Ecuador) respecto a la experiencia del área de conservación privada Chaparrí (Perú)}

Vol. 3, núm. 3., (2019)

María José Ortiz Zurita; Jazmín Jessenia Peñafiel León; Gabriela Natalia Torres Jara; Diana Valeria Delgado Campuzano; Evelyn Mariuxi Bautista Guaranda

constituirse como Área de Conservación firmando convenios y estableciendo los objetivos que se querían lograr, entre ellos el principal era ofertar un producto turístico considerando que es una actividad que permite incorporar la conservación tanto del entorno como de las especies.

Asimismo, el Área de Conservación Privada Chaparrí se encuentra muy apegada a la conservación de especies propias del sector entre sus proyectos de conservación podemos mencionar los siguientes: centro de rescate y rehabilitación para osos Andinos, siendo la única en todo Perú, reintroducción de la pava aliblanca lo que ha ayudado a que actualmente se repueblen naturalmente, reintroducción del guanaco camélido más grande de Sudamérica, recuperación del Cóndor Andino, se encuentran trabajando en la introducción de nuevos ejemplares y en un programa de oferta de alimento suplementario. Para poder ingresar al Área de Conservación Privada Chaparrí se debe hacer el pago de \$3,06 si solo se trata de un full day, si es el caso de pernoctación el valor es de $\$ 9,16$ lo cual corresponde al permiso por ingreso.

Otro aporte de relevancia, es que la reserva es manejada por la comunidad muchos de ellos han empezado con emprendimientos que sirven como actividades complementarias a la oferta de turismo como cuatrimotos para realizar recorridos en áreas cercanas a la población, otro comunero está implementando áreas de camping, entre otros. Tiene temporadas altas que van de mayo a septiembre y temporadas bajas en los meses de Junio - Agosto y Enero - Abril. De manera general la reserva ha presentado un ligero incremento de turistas extranjeros $\mathrm{y}$ decrecimiento en el mercado nacional, en cuanto al primer mercado se debe a que han estado presentes esporádicamente en ferias como la de Rutland en Inglaterra, y en cuanto a su mercado 


\section{Estudio conservacionista del bosque protector cerro Blanco (Ecuador) respecto a la experiencia del área de conservación privada Chaparrí (Perú)}

Vol. 3, núm. 3., (2019)

María José Ortiz Zurita; Jazmín Jessenia Peñafiel León; Gabriela Natalia Torres Jara; Diana Valeria Delgado Campuzano; Evelyn Mariuxi Bautista Guaranda

nacional principalmente viene por parte de colegios, universidades, delegaciones de institutos, empresas, entre otros que generalmente realizan visitas de un día.

Entre las especies presentes dentro del Área se encuentran registradas 65 aves y 9 especies de mamíferos de las cuales 21 y 9 especies están consideradas en peligro a nivel mundial respectivamente, además hasta el $60 \%$ de anfibios y reptiles de esta región son endémicos. Sus objetivos, están relacionados a los pilares fundamentales del ecoturismo como los siguientes: Desarrollar el concepto de que las comunidades deben crear sus propios proyectos que les permita el desarrollo sostenible en el tiempo sin una ayuda permanente de mano extranjera, Preservar el ecosistema y hacer uso responsable del mismo para el desarrollo de actividades económicas que permita generar ingresos y mejorar la calidad de vida de los comuneros, Informar al público en general de las actividades que se han desarrollado, se desarrollan y se plantean desarrollar dentro de la Reserva.

Finalmente se puede acotar, que dicha área de conservación le ofrece al turista en general, oportunidades de esparcimiento, descanso, recreación y al mismo tiempo elevar su conciencia ambientalista con miras a buscar mejores acciones dirigidas a la conservación del turismo arqueológico, como fuente de ingreso en el Perú. Por lo tanto, cada una de estas fortalezas, son consideradas elementos esenciales para la construcción de la propuesta orientada a un manejo eficiente del Bosque Protector Cerro Blanco ubicado en Ecuador. 


\section{Estudio conservacionista del bosque protector cerro Blanco (Ecuador) respecto a la experiencia del área de conservación privada Chaparrí (Perú)}

Vol. 3, núm. 3., (2019)

María José Ortiz Zurita; Jazmín Jessenia Peñafiel León; Gabriela Natalia Torres Jara; Diana Valeria Delgado Campuzano; Evelyn Mariuxi Bautista Guaranda

Propuesta

Título

Propuesta de Manejo para el Bosque Protector Cerro Blanco (Ecuador) en base al estudio del Área de Conservación Privada Chaparrí (Perú)

\section{Justificación}

Cada una de las acciones realizadas durante la recolección de información e interpretación de la misma, permite indicar que la realización de esta propuesta, se justifica primeramente por la presencia de diferentes situaciones negativas encontradas en la actuación ciudadana en relación al uso de la tala como actividad productiva que destruye las riquezas presente en esta zona protegida, en segundo término se puede evidenciar que el gobierno ecuatoriano ha realizado diferentes acciones legales con el fin de garantizar su conservación como un patrimonio cultural y ambiental; pero las mismas, en ciertas condiciones presenta poca actuación al mantener en la zona actividades de explotación que reducen la biodiversidad del mismo.

De igual manera, se justifica por una fortaleza de naturaleza económica que permiten reconocer que el Bosque Protector de Cerro Blanco presenta diferentes elementos que conforman el paisaje, tales como las condiciones climáticas, características del relieve, potencialidad del suelo, estructura de la vegetación y fauna. A través de dicho análisis se logra identificar los procesos o cambios que en un momento dado pueden alterar el funcionamiento de un sistema 


\section{Estudio conservacionista del bosque protector cerro Blanco (Ecuador) respecto a la experiencia del área de conservación privada Chaparrí (Perú)}

Vol. 3, núm. 3., (2019)

María José Ortiz Zurita; Jazmín Jessenia Peñafiel León; Gabriela Natalia Torres Jara; Diana Valeria Delgado Campuzano; Evelyn Mariuxi Bautista Guaranda

ambiental y poder llevar a cabo nuevas acciones mediante las cuales se puedan agregar cambios progresivos que beneficien a todos los elementos existente en dicha zona protegida.

Un aspecto de interés que justifica el plasmar la propuesta para el manejo del Bosque Protector Cerro Blanco, lo constituye el encontrarse como otros bosques de Guayaquil rodeado por urbanizaciones, canteras y carreteras y esto no permite el flujo genético de las especies. A pesar de los constantes monitoreos por parte de guarda parques existe una latente preocupación por el ingreso de personas a realizar actividades de tala y caza ilegal. Además, se puede resaltar que el bosque por ser propiedad de la compañía Holcim Ecuador, administrado por la Fundación Pro-Bosque, permite que la mayor parte del bosque protector está destinada a la conservación de la diversidad biológica, sin embargo existen áreas donde se realiza ecoturismo y actividades de reforestación.

De las consideraciones anteriores, se puede visualizar que el área de Conservación Privada Chaparrí (Perú), por representar grandes potencialidades, se convierte en un referencial significativo para plasmar las respectivas acciones que ayuden a la Fundación Pro-Bosque a la realización de nuevas experiencias como parte esencial para conservar y preservarlo como una riqueza ambiental de alta representación para la nación (Ecuador).

\section{Fundamentación}

El objetivo principal del Bosque Protector Cerro Blanco (BPCB) es Proteger y rehabilitar una muestra representativa de importancia nacional de la región del bosque seco tropical, fomentando la comprensión, apreciación y deleite público para no destruirlo y guardarlo para las 


\section{Estudio conservacionista del bosque protector cerro Blanco (Ecuador) respecto a la experiencia del área de conservación privada Chaparrí (Perú)}

Vol. 3, núm. 3., (2019)

María José Ortiz Zurita; Jazmín Jessenia Peñafiel León; Gabriela Natalia Torres Jara; Diana Valeria Delgado Campuzano; Evelyn Mariuxi Bautista Guaranda

generaciones presentes y futuras, tanto de ecuatorianos como visitantes internacionales. Para analizar la sustentabilidad de la Zona de Recreación al Aire Libre del BPCB. La implementación del diseño de los nuevos senderos para el BPCB implica la elaboración de todos aquellos accesorios o construcciones necesarias para el buen desarrollo del mismo. El objetivo final es evaluar alternativas para implementarlas en el lugar considerando la normativa técnica, ambiental y turística.

En este sentido, Ortiz, Penafiel, Torres, Delgado y Bautista (ob.cit), precisan que la conservación y protección de la naturaleza es un tema que ha despertado gran interés a nivel mundial, y la creación de áreas protegidas ha permitido resguardar los recursos naturales y culturales de muchos sitios que los poseen en mayor número. El objetivo principal del Bosque Protector Cerro Blanco $(\mathrm{BPCB})$ es proteger y rehabilitar una muestra representativa de importancia nacional de la región del bosque seco tropical, fomentando la comprensión, apreciación y deleite público para no destruirlo y guardarlo para las generaciones presentes y futuras, tanto de ecuatorianos como visitantes internacionales.

\section{Contenido de la Propuesta}

La suma de los elementos combinados en materia de conservación y protección permite tomar en consideración las siguientes acciones a incorporar como medidas que ayuden a resguardar al Bosque Protector Cerro Blanco, mediante actividades conjuntas de sus miembros. 


\section{Estudio conservacionista del bosque protector cerro Blanco (Ecuador) respecto a la experiencia del área de conservación privada Chaparrí (Perú)}

Vol. 3, núm. 3., (2019)

María José Ortiz Zurita; Jazmín Jessenia Peñafiel León; Gabriela Natalia Torres Jara; Diana

Valeria Delgado Campuzano; Evelyn Mariuxi Bautista Guaranda

Evaluar de manera colaborativa el contenido que caracteriza al plan maestro del Área de Conservación Chaparrí (Perú), con el fin de promocionar entre los grupos la realización de mesas de trabajo determinadas por valorar las necesidades económicas presente, para así, estimar posibles cambios en cuanto a los ingresos y aumentar la presencia de trabajadores encargados de velar por su protección y vigilancia.

Ofrecer a los trabajadores del Bosque Protector Cerro Blanco, actividades de capacitación, mediante ellas adecuar un entrenamiento que les ayude a mejorar las relaciones interpersonales con los turistas en pro de incrementar su asistencia como parte de una vivencia ambiental que fomente durante la realización de las actividades ambientales la formación de una conciencia centrada en la corresponsabilidad de preservar y velar por el cuidado de dicha zona protegida, en otras palabras, donde la comunidad sea un agente motivador de las mismas.

Como algo sucesivo al primer punto se propone establecer un circuito combinado de avistamiento de aves entre el Bosque Protector Cerro Blanco y la comunidad Puerto Hondo, dando a conocer las diferentes especies existentes en estos dos diferentes tipos de entornos.

Revisar el marco legal, con el fin de lograr mediante el desarrollo de acciones administrativas-jurídico orientar el uso de la tierra en actividades tanto de conservación como el manejo sustentable de los recursos, de esta manera, los programas de conservación deben apuntar a un manejo sostenible para asegurar ingresos constantes a la comunidad, siendo manejados por elementos propios de la comunidad. 


\section{Estudio conservacionista del bosque protector cerro Blanco (Ecuador) respecto a la experiencia del área de conservación privada Chaparrí (Perú)}

Vol. 3, núm. 3., (2019)

María José Ortiz Zurita; Jazmín Jessenia Peñafiel León; Gabriela Natalia Torres Jara; Diana Valeria Delgado Campuzano; Evelyn Mariuxi Bautista Guaranda

Desarrollar actividades de investigación que permitan mantener muestras relacionadas con el ecosistema del Bosque Protector Cerro Blanco, evento que dará cabida a la continuidad de programas dirigidos a la conservación para el mantenimiento de los procesos ecológicos y las respectivas especies de flora y fauna que pudieran estar amenazadas dentro del lugar.

Buscar mecanismos de enlaces con las diferentes empresas turistas que hacen vida en Ecuador, a fin de lograr mediante su intervención la respectiva promoción de las características ambientales, valores culturales y patrimonio nacional, esta acción promocionará el incremento de los turistas; quienes se convertirán en multiplicadores de vivencias en cuanto a las diferentes actividades promocionadas en el Bosque Protector de Cerro Blanco (Ecuador).

Establecer contactos con las diferentes instituciones educativas en los diferentes niveles a fin de promocionar visitas que ayuden a los estudiantes a generar el respectivo contacto con la naturaleza, los valores ambientales, culturales y puedan reconocer la importancia de preservar el patrimonio natural de su país.

\section{Conclusiones.}

Según la interpretación desarrollada a lo largo del cuerpo teórico que caracteriza al artículo, se pueden considerar los siguientes argumentos:

Las actividades vinculadas con el ecoturismo, representa para Ecuador una fuente de ingreso, razón por la cual, es importante elevar su atención hacia la conservación y preservación de los espacios naturales o zonas protegidas, con el fin de garantizarle a las nuevas generaciones 


\section{Estudio conservacionista del bosque protector cerro Blanco (Ecuador) respecto a la experiencia del área de conservación privada Chaparrí (Perú)}

Vol. 3, núm. 3., (2019)

María José Ortiz Zurita; Jazmín Jessenia Peñafiel León; Gabriela Natalia Torres Jara; Diana Valeria Delgado Campuzano; Evelyn Mariuxi Bautista Guaranda

el disfrute de conocerlas y despertar al mismo tiempo la conciencia ambientalista como respuesta de valoración de los ambientes naturales con todos sus elementos.

Los procesos relacionados con la conservación y manejo de las zonas protegidas se ha convertido hoy en día, en una mecánica capaz de resolver los daños ocasionados a los ecosistemas naturales, además, reduce la posibilidad de extinción de las especies esenciales para la vida humana. Asimismo, se puede resaltar que, cuando las naciones cuentas con zonas protegidas es inminente la intervención del gobierno, instituciones, organizaciones entre otros medios que permitan garantizar el rescate de los valores ambientales como pilares para una sociedad justa.

Mantener en el trabajo mancomunado con la comunidad y otros países potenciales en materia de conservación ambiental, a fin de establecer en el Bosque Protector Cerro Blanco, nuevas actuaciones cónsonas con las necesidades que allí se presentan y en función de ello, garantizar beneficios generales para el respectivo bosque. Otro aspecto, de importancia lo constituye el trabajo de expertos en materia ambiental en pro de crear actividades de intercambio para afianzar un manejo eficiente que ofrezca respuestas oportunas a todos los recursos existentes, así como, al turista y comunidad en general.

\section{Bibliografía.}

Arias, F. (2011). El Proyecto de Investigación. Caracas: Episteme .

Barrientos, C. (2019). Análisis Elemental de Encuestas. México: Trillas.

Medina Villacrés, J. (2018). El ecoturismo en Ecuador: Actualidad y perspectivas. Revista Observatorio de la Economía Latinoamericana, 3(3), 1-10. 


\section{Estudio conservacionista del bosque protector cerro Blanco (Ecuador) respecto a la experiencia del área de conservación privada Chaparrí (Perú)}

Vol. 3, núm. 3., (2019)

María José Ortiz Zurita; Jazmín Jessenia Peñafiel León; Gabriela Natalia Torres Jara; Diana Valeria Delgado Campuzano; Evelyn Mariuxi Bautista Guaranda

Ortiz Zurita, M., Peñafiel León, J., Torres Jara, G., Delgado Campuzano, D., \& Bautista Guaranda, E. (2019). Propuesta de manejo para el Bosque Protector Cerro Blanco (Ecuador), en base al estudio del área de Conservación Privada Chaparrí (Perú). RECIAMUC, 3(2), 1236-1253.

Paz, B. (2019). Recorre la Naturaleza. España: Narcea .

Paz, H. (2019). Bosque Cerro Blanco. Ambente, 25-36.

Pérez, H. (2019). Introducción a los Métodos Cualitativos. Barcelona: Paidós. 